\title{
Méthodes traditionnelles de lutte contre les insectes et les tiques chez les Mossé au Burkina Faso
}

\author{
${ }^{1 *}$ Salfo SAVADOGO, 3,2 Oumarou SAMBARE, ${ }^{1}$ Abdoulaye SEREME, ${ }^{2}$ Adjima THIOMBIANO \\ ${ }^{1}$ Departement Substances Naturelles, Institut de Recherche en Sciences Appliquées et Technologies, Centre National de \\ la Recherche Scientifique et Technologique, 03 BP 7047 Ouagadougou 03, Burkina Faso ; Fax : (226)25357029 \\ 2Laboratoire de biologie et écologie végétales, Unité de Formation et de Recherche en Sciences de la Vie et de la Terre, \\ Université de Ouagadougou, 09 BP 848 Ouagadougou 09 Burkina Faso. \\ 3Institut Des Science (IDS), 01 BP 1757 Ouagadougou 01 Burkina Faso. \\ *Auteur correspondant: Salfo SAVADOGO : (226) 78148774/ (226) 76091008; Email : asalfosava@yahoo.fr I \\ salfosava@gmail.com
}

Original submitted in on $11^{\text {th }}$ July 2016. Published online at www.m.elewa.org on 30 th September 2016 http://dx.doi.org/10.4314/jab.v105i1.9

\section{RÉSUMÉ}

Objectif : L'objectif de cette étude est de connaître les techniques endogènes de lutte contre les pestes et les usages que les populations font des plantes en société Mossé au Burkina Faso.

Méthodologie et résultats : La présente étude s'est basée sur des interviews auprès des populations locales. Les données des enquêtes ont été saisies puis traitées sur Excel 2010 et sur JMP 8 au seuil de 0,05. II ressort des enquêtes que les populations utilisent des plantes pour lutter contre les insectes et les tiques dans la conservation des graines de céréales et de légumineuses, les parasites des animaux et les insectes vecteurs de maladies. Vingt-quatre (24) espèces au total ont été recensées. En plus des plantes locales, les populations utilisent l'exposition au soleil, l'enfumage, des matières inertes et la conservation en atmosphère confinée.

Conclusion et application : Cette étude a permis de connaître les types d'usages traditionnels que les Mossé font des plantes. Ces techniques traditionnelles permettraient de faire l'économie des méthodes modernes, en l'occurrence les méthodes chimiques qui ont déjà montré leurs effets sur l'environnement ; d'où la nécessité de promouvoir et de valoriser ces méthodes traditionnelles.

Mots clés : Savoirs locaux, espèces végétales, pestes, interview, Mossé, Burkina Faso

\begin{abstract}
Traditional methods of fight against insects and ticks in Mossé society (Burkina Faso)

Objective: The objective of this study is to know the endogenous control techniques against pests and the traditional uses of plants in Mossé society in Burkina Faso.

Methodology and Results : The study is based on interviews with local people. Survey data were entered and processed on Excel 2010 and JMP 8 at the 0.05 level. The investigations allowed to show that the populations use plants for various ends such as the conservation of cereal seeds and leguminous plants, the fight against insects responsible of diseases, parasites of the animals. Twenty-four (24) species were listed. In addition to the local plants, the populations use the exposure to the sun, smoking, the inert matter and the conservation in air confined.
\end{abstract}



les Mossé au Burkina Faso

Conclusion and Application : This study showed the types of traditional uses of plants in Mossé society. These traditional techniques would do without modern methods, namely chemical methods which have already shown their effects on the environment ; hence the need to promote and enhance these traditional methods.

Key words : local knowledge, plants, pests, interview, Mossé, Burkina Faso

\section{INTRODUCTION}

Au Burkina Faso, le secteur primaire, constitué par l'agriculture et l'élevage emploie presque toute la population rurale et une partie importante de la population urbaine. Entre 80 et $85 \%$ des burkinabè travaillent dans le secteur primaire (Ministère de l'Économie et des Finances du Burkina Faso, 2010). Cependant, le faible rendement agricole dû aux méthodes archaïques et aux aléas climatiques rendent cette partie de la population pauvre et fragile même si elle contribue à hauteur de $40 \%$ au petit PIB (Ministère de l'Économie et du Développement, 2006 ; Ministère de l'Économie et de Finances du Burkina Faso, 2010). Le climat et les traditions conditionnent les activités agricoles qui sont réparties entre les différentes communautés du pays et entre les différentes régions. L'élevage est lui aussi archaïque et fragile. L'année 2006 a vu mourir de nombreuses têtes de troupeaux dans le nord faute de pâturages et d'eau en raison de l'hivernage tardif (Ministère de l'Économie et du Développement, 2006). Les maladies n'épargnent pas non plus les bêtes : la peste porcine décime les cochons et chaque année les grippes aviaires déciment une grosse partie des élevages avicoles traditionnels (Ministère de l'Économie et du Développement, 2006). Confinements, vaccinations et préventions sont totalement absents des usages chez des éleveurs n'ayant généralement aucune formation de base. II est de nécessité impérieuse de trouver des voies et moyens pour contrecarrer toutes ces menaces et particulièrement celles des pestes sur une agriculture déjà sous le poids de la précarité du climat. Les pestes sont des organismes animaux, végétaux ou de microorganismes nuisibles à la vie d'autres plantes, animaux ou microorganismes. Ces pestes causent des dommages très considérables dans le domaine de l'agriculture, de l'agroforesterie, de l'élevage, de la santé (Akpavi et al. 2007; Noémie, 2010 ; Delimi et al., 2013 ; Baerts et al., $\mathrm{SD})$. Les nouvelles problématiques du changement social et économique dans le monde prônent la participation des acteurs dits locaux. Au Burkina Faso, la recherche agronomique qui est au centre de la création et de l'évaluation des technologies s'efforce de développer des approches favorisant une meilleure compréhension des systèmes de production (Sankara et Namono, 2004). L'intensification de la production agricole n'est pas envisageable sans l'adoption de technologies appropriées dont la lutte contre les ravageurs des cultures (Ouédraogo, 2005 ; Bambara et Tiemtoré, 2008). En effet, les populations des organismes nuisibles augmentent de façon considérable, ce qui augmente la gravité des dommages infligés aux cultures (Pintureau, 2006 ; Habiba, 2007; Guilli et al., 2009). Les interventions phytosanitaires ont jusqu'à présent contribué à des degrés divers au contrôle des ennemis des plantes et des animaux (Sigala, 2001 ; Akpavi et al., 2007). Depuis longtemps, la lutte contre ces ennemis et en particulier ceux des cultures est basée sur l'utilisation des pesticides de synthèse. L'usage de ces pesticides chimiques a souvent causé beaucoup plus de problèmes qu'il n'en a résolu (El Guilli et al., 2009 ; Chandrashekar et Srinivasa, 2003). Selon Ouédraogo (2004), cette utilisation des pesticides de synthèse pose les problèmes majeurs suivants: (i) l'accroissement de la résistance des insectes, (ii) la disparition des populations d'insectes, (iii) la pollution des eaux de surface et des nappes phréatiques, (iv) la neutralisation de la vie du sol, (v) l'Afrique utilise moins de $10 \%$ de la production mondiale de pesticides mais totalise $75 \%$ des cas mortels dus aux pesticides, etc. En outre, les technologies proposées n'ont pas toujours eu le succès escompté pour plusieurs raisons dont leur inadéquation (Guèye et al., 2011), les risques associés à leur adoption (Regnault-Roger, 2002), l'accoutumance des insectes et la sélection de souches résistantes (Benhalima et al., 2004), la pauvreté, etc. C'est pourquoi, aujourd'hui, pour des raisons écologiques et économiques, il y a nécessité de développer des 
méthodes de substitution aux pesticides de synthèse dans la protection des cultures. Parmi ces méthodes, les biopesticides occupent une place de choix (Ouédraogo, 2004). La présente étude se propose de répertorier toutes les connaissances traditionnelles en matière de lutte contre les pestes à des cultures et des animaux. Elle a pour objectif de connaître les techniques endogènes de lutte contre les pestes et les différents usages que les

\section{MATERIEL ET METHODES}

Échantillonnage et collecte de données: Des enquêtes qualitatives et participatives, basées sur des interviews semi-structurées ont été conduites dans trente (30) villages appartenant à quatre (4) provinces que sont populations font des plantes en société Mossé. II s'agira plus spécifiquement de :

-recenser toutes les plantes à potentialité biopesticides/biopestifuges et leurs domaines d'utilisation ;

-répertorier toutes les méthodes et techniques traditionnelles en matière de lutte contre les pestes.

le Yatenga, le Kourweogo, le Kadiogo et l'Oubritenga (figure 1). Ces provinces relèvent de trois régions administratives à savoir les régions du nord, du centre et du plateau central.

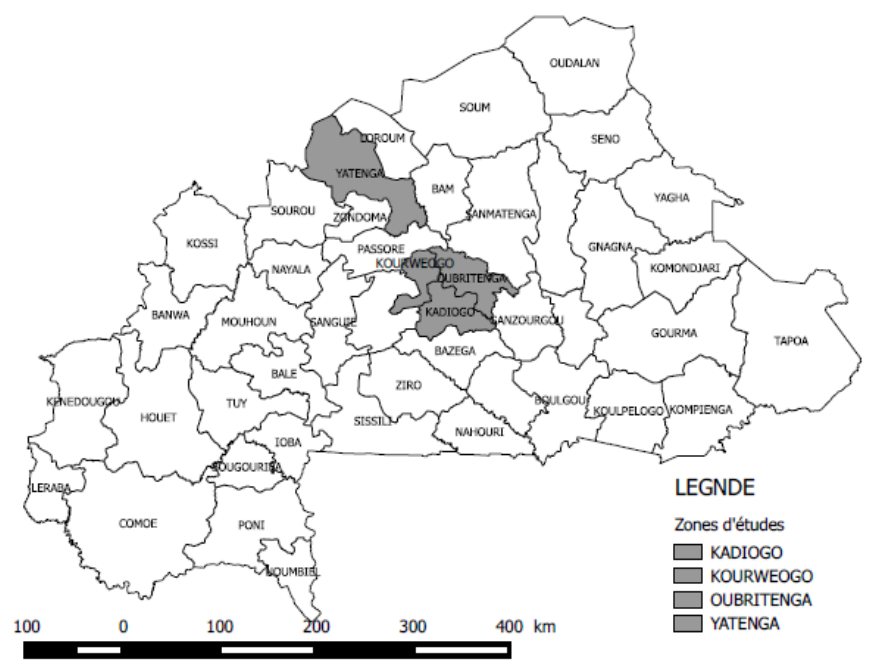

Figure 1 : Localisation de la zone d'étude

Les différentes localités ont été choisies sur la base de la vivacité de leurs pratiques culturelles. En effet, à l'issue d'enquêtes préliminaires, il est ressorti que dans ces quatre (4) provinces, les populations ont longtemps conservé leurs pratiques en matière de lutte contre les pestes et de conservation des semences. Dans chaque localité, les personnes des deux sexes ont été soumises à un questionnaire. Les autorités coutumières ont été ciblées, les chefs d'exploitation et leur(s) femme(s) ainsi que des responsables de divers services agricoles de la zone ont été les répondants. Le nombre moyen de personnes par focus groupe était de 15. L'enquête s'est déroulée avec les paysans à domicile. Ensuite, nous nous sommes déplacés dans les champs et marchés pour collecter des échantillons de plantes citées pendant l'entretien. Les plantes cueillies ont servi à constituer un herbier. Le choix des localités était guidé par les autorités coutumières et d'autres personnes d'âges avancés. Cependant, nous avons jugé nécessaire de prendre en compte certaines localités voisines qui n'ont pas été recommandées par ces autorités. Cela répond aux soucis d'obtenir des données représentatives et plus fiables, considérant les risques éventuels d'omission et d'ignorance de la part des autorités (Savadogo, 2013). Le questionnaire a porté sur les différentes utilisations des espèces à des fins biopesticides ou biopestifuges, les 

les Mossé au Burkina Faso

types de pestes sur lesquels ces plantes sont utilisées, les méthodes utilisées autres que les plantes locales, pour lutter contre les pestes. Les pratiques agricoles de conservation des récoltes ont été également notées selon leur modalité, leur opportunité et leur efficacité. Il s'est agi d'observer et de décrire objectivement ce que le paysan fait, d'analyser la logique interne ou la rationalité de ses pratiques. L'entretien individuel n'est pas adapté dans cette étude en ce sens que les enquêtés refusaient de nous donner des informations quand ils sont seuls, de peur de faillir à la tradition en diffusant les secrets du village. Même les chefs coutumiers lorsqu'ils sont seuls, n'acceptent toujours pas de répondre à nos interrogatoires. Ils préfèrent réunir leurs notables ou autres personnes d'âges avancés, avant de nous donner des informations. Pour avoir plus de crédibilité aux réponses données par les enquêtés, nous avons privilégié les entretiens en groupe. En effet, une seule personne n'est pas censée avoir des connaissances sur certains aspects et, pourrait nous fournir des informations biaisées. Par contre, dans les entretiens en groupe, les réponses sont progressivement corrigées et réajustées par certaines personnes lorsqu'on constate des imprécisions ou des incorrections (Savadogo, 2013). Les

\section{RESULTATS}

Domaines d'utilisation des espèces végétales : Les enquêtes révèlent que les espèces végétales sont utilisées dans trois (03) domaines pour lutter contre les insectes et les tiques. Ce sont: le domaine de la conservation des céréales (Pennisetum americanum, Sorghum bicolor, Zea mays) et des légumineuses (Vigna unguiculata, Voandzeia subterranea), dans le domaine de la santé humaine et animal (la lutte contre les insectes, agents vecteurs de maladies diverses) et dans le entretiens se sont déroulés en langues locales, avec l'aide d'interprètes. Les noms des plantes, cités en langues locales, sont identifiés par leurs noms scientifiques sur le terrain ou le cas échéant, des échantillons d'herbier ont été collectés pour être déterminés au laboratoire de l'Herbier de l'Université Ouaga I Pr Joseph KI-ZERBO. La nomenclature utilisée est celle de International Plant Names Index data base (IPNI, 2011). Lorsque la plante citée n'existe plus dans les terroirs étudiés, son nom local est retenu pour être identifié avec l'appui des systématiciens qui parle la langue ou par la littérature existante.

Traitement des données : Le dépouillement des guides d'entretien a été réalisé manuellement. A l'aide de Microsoft EXCELC, les données ont servi à calculer le pourcentage d'adoption des pratiques par les paysans, c'est-à-dire le pourcentage de paysans qui adoptent une pratique et la mettent effectivement en œuvre. Ce qui a permis d'estimer l'importance relative de chaque pratique. Ces analyses ont permis de construire des histogrammes illustratifs pour les différents domaines d'utilisation des plantes. Les analyses de variances sur les effectifs des enquêtés par localité ont été réalisées sur JMP 8 au seuil de $5 \%$.

domaine de la lutte contre les parasites des animaux (figure 2). Les espèces utilisées pour la conservation des céréales et des légumineuses sont les plus citées $(63,33$ $\%)$ suivis de très loin des espèces utilisées contre les insectes $(43,33 \%)$. Les espèces utilisées contre les parasites des animaux ne représentent que 12,66 \% (figure 2). II n'y a pas de différences significatives entre les effectifs des enquêtés par localité $(p=0,42)$. 


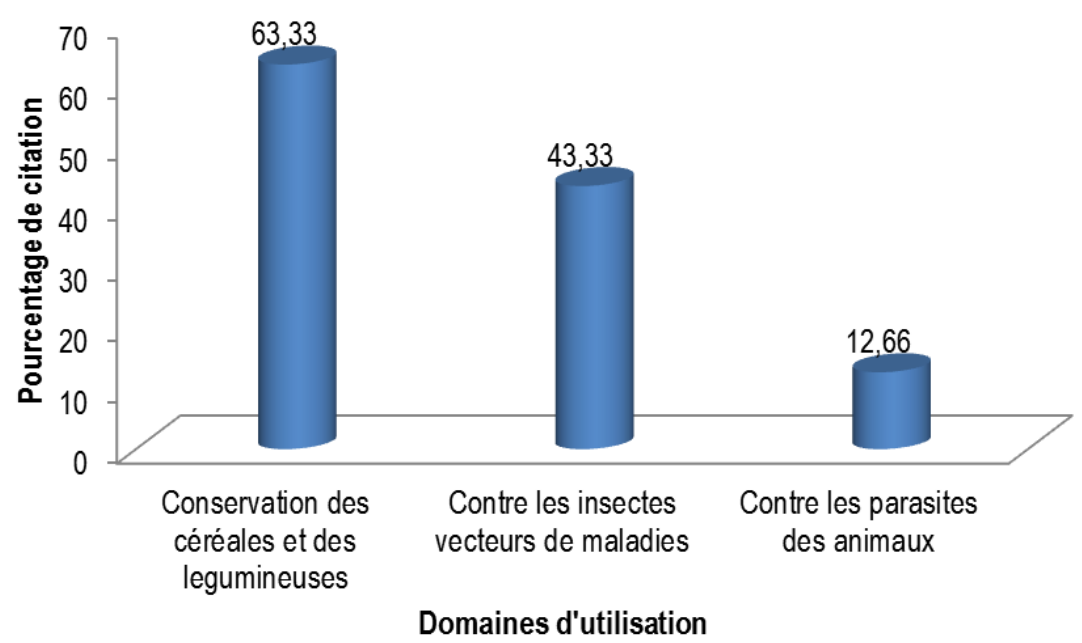

Figure 2 : Domaines d'utilisation des espèces et leur pourcentage de citation

Espèces végétales et techniques de conservation des céréales et des légumineuses: Au total douze (12) espèces végétales appartenant à 10 familles et 11 genres sont citées dans le domaine de la conservation des céréales et des légumineuses. Les espèces les plus citées sont: Hyptis spicigera (95,52\%), Azadirachta indica $(41,27 \%)$, Cymbopogon schoenanthus $(38,27 \%)$, Ocimum americanum $(31,4 \%)$ et Hyptis suaveolens $(28,32 \%)$ (Figure 3 ).

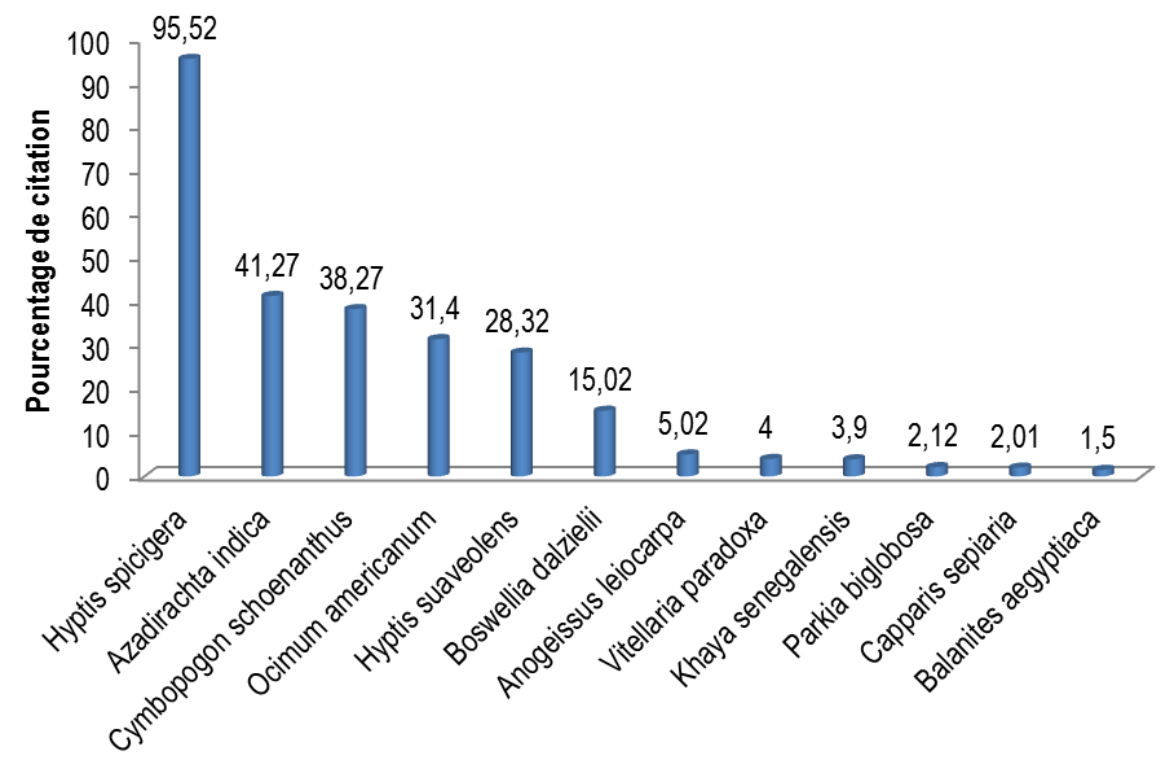

Espèces

Figure 3 : Pourcentage de citation des espèces dans le domaine de la conservation des céréales et des légumineuses

Les cendres potassées de Anogeissus leiocarpa, de Khaya senegalensis et de Vitellaria paradoxa sont très efficaces pour la conservation des graines de Niébé et du pois de terre. Ces cendres sont mélangées avec les dites graines et conservées dans des greniers en banco, dans des bidons ou dans des barriques métalliques. En générale, la durée maximale de conservation est d'une (01) année. 8,5\% des enquêtés continuent d'utiliser cette 
méthode de conservation des semences. Les feuilles de Hyptis spicigera, Hyptis suaveolens, Ocimum americanum, les feuilles et les inflorescences de Cymbopogon schoenanthus sont mélangées aux gousses de niébé ou du pois de terre de façon étagées avant d'être conservées dans des greniers construits en chaumes. L'huile des graines de Azadirachta indica est aspergée sur les graines en conservation tandis que les feuilles sont séchées puis broyées à la main avant d'être mélangées aux graines du niébé, du sorgho et du pois de terre. Si pour beaucoup de paysans ces méthodes traditionnelles de conservation ne nécessitent aucun rituel, pour d'autres, ces méthodes pour être très efficaces et effectives, elles doivent être accompagnées de rituels. C'est le cas pour les habitants de Tanseiga, dans la commune rurale de Komsilga (province du Kadiogo) pour qui la conservation des graines du pois de terre ne doit pas se faire n'importe comment et n'importe quand. Pour ces habitants, au moment de la conservation des graines du pois de terre, du niébé et autres semences, l'operateur doit d'abord tamiser les cendres pour les débarrasser de leurs impuretés et, se lever le premier jour du mois lunaire ou le 8 eme jour très tôt le matin sans parler à quelqu'un faire tout le travail et déposer le mélange cendre- niébé ou pois de terre dans un endroit inaccessible à d'autres personnes. Par ailleurs, d'autre pensent que l'opératrice ne doit pas être une femme en état d'impureté majeur, c'est-à-dire en période de menstrues. La non observance de toutes ces règles conduirait, aux dires de certains des enquêtés, à brève échéances à la destruction des graines par les insectes. Pour la conservation du niébé, ces habitants utilisent également le mélange feuilles de Ocimum americanum plus celles de Hyptis spicigera. Une première couche constituée de ces feuilles est d'abord déposée au fonds du grenier en chaume avant de placer les gousses du niébé. Une dernière couche est en fin placée au-dessus de ses gousses avant de fermer le grenier. Les paysans luttent contre les termites en utilisant les cendres de n'importe quelle espèce. Pour ce faire, le matériel à conserver est saupoudré de cendre avant d'être déposé dans un endroit isolé. Si le matériel à conserver ou à préserver est destiné à être déposé au sol, le sol est d'abord saupoudré par les cendres avant de placer le matériel à conserver. La potasse en poudre est très souvent appréciée par beaucoup de femmes pour la conservation des graines de pois de terre. Dans cette pratique, les graines sont badigeonnées de potasse en poudre puis homogénéisées avant d'être conservé dans des récipients quelconques. Certaines femmes estiment que cette méthode permet une conservation des graines pendant une période de cinq (05) ans. D'autre préfèrent les bulbes d'oignons pour la conservation des graines de sorgho. A cet effet, les bulbes d'oignon sont mis dans des sachets en plastiques bien attachés et enfui dans les graines de mil. Cela permet une meilleure conservation du mil pendant une longue période. Pour une meilleure conservation des graines de sorgho, d'autres paysans mélangent ces graines à celles de Balanites aegyptiaca. Ces dernières seraient très efficaces en matière de conservation selon certains enquêtés. Certaines femmes estiment que les feuilles pilées de Capparis sepiaria peuvent être utilisées pour la conservation de n'importe quelle semence à cause de son goût amer. Les feuilles de mil pilées seraient par contre indiquées pour la conservation du sorgho.

Espèces végétales et techniques de lutte contre les insectes (agents vecteurs des maladies) : Certaines espèces ont des propriétés insectifuge où insecticide et sont de ce fait utilisées par la population dans la lutte contre les insectes. Les quatorze (14) espèces recensées pour cette cause se répartissent en neuf (09) familles et treize (13) genres. Elles représentent $13,33 \%$ des espèces citées par l'ensemble de nos enquêtés. Au nombre de ces espèces on peut citer: Hyptis spicigera $(75,61 \%)$, Azadirachta indica $(68,7 \%)$, Vitellaria paradoxa $(47,35 \%)$, Ocimum americanum (41,36\%), Hyptis suaveolens (40,17\%), Eleusine indica (38,47\%), Zea mays (35,9\%), Solanum incanum (32,8 \%), Boswellia dalzielii $(27,2 \%)$, Cyanotis lanata $(23,9 \%)$, Cymbopogon schoenanthus (22,2 \%) (Figure 4). 


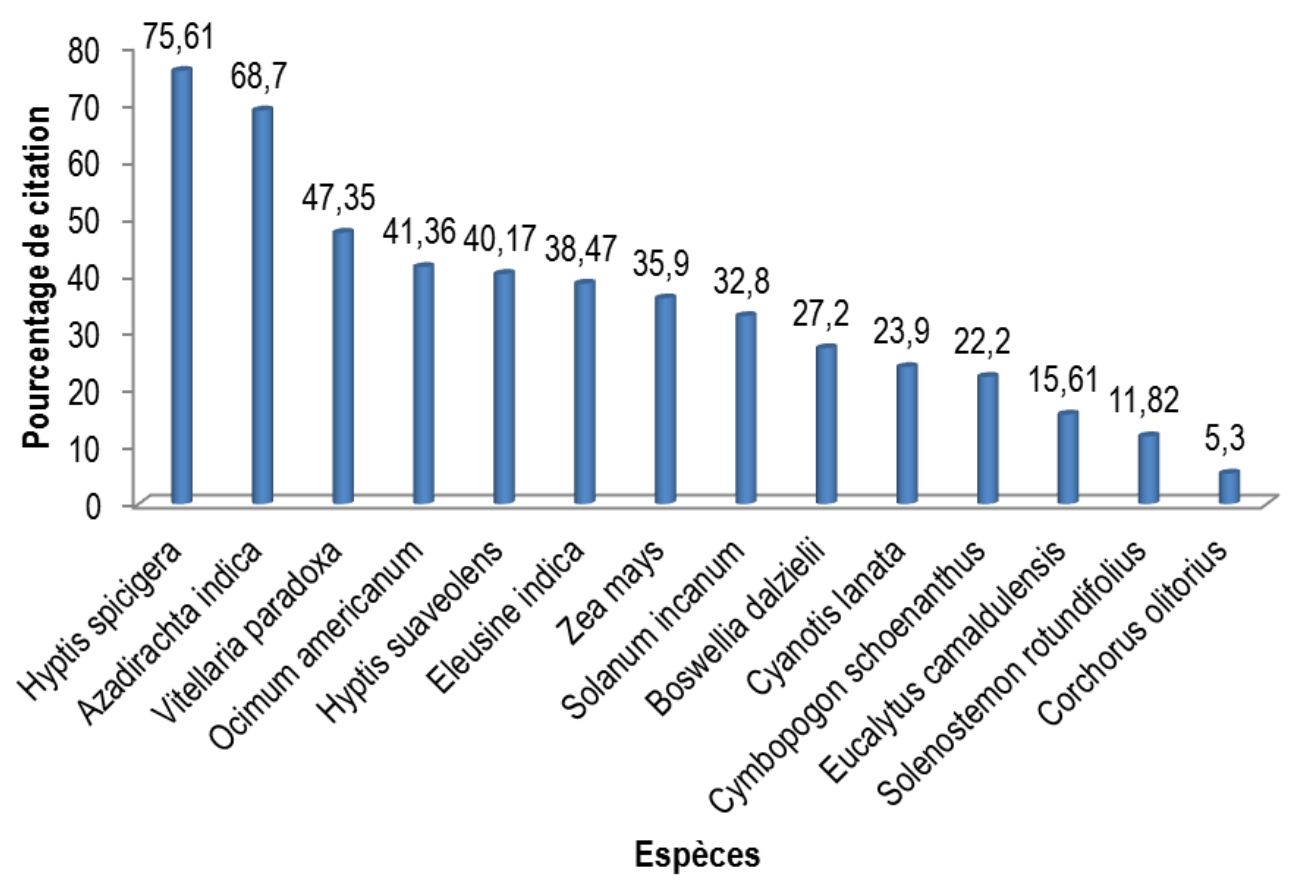

Figure 4 : Pourcentage de citation des espèces dans la lutte contre les insectes vecteurs de maladie

De la sève sèche de Boswellia dalzielii brulée dans un récipient dégage une fumée insectifuge. Cette méthode est pratiquée par $5 \%$ de nos enquêtés. Les feuilles (fraiches ou sèches) de Azadirachta indica présentent également les mêmes effets. D'autres habitants utilisent les coques des amandes de Vitellaria paradoxa en association avec les épis de Zea mays dépourvus de leurs graines. La fumée de ce mélange est réputé être efficace pour chasser les moustiques. La patte obtenue après la fabrication du beurre de karité est séchée puis utilisée contre les moustiques vecteurs de maladies. Cette poudre obtenue après séchage est incinérée, la fumée qui se dégage est hautement insectifuge par son odeur très caractéristique. Pour chasser les moustiques, les habitants de Tanseiga utilisent le mélange feuilles de Eleusine indica plus les coques des amandes du karité. $\mathrm{Ce}$ mélange placer dans un récipient puis bruler dégagerait de la fumée nocive aux moustiques. L'odeur de Hyptis suaveolens serait également un puissant insectifuge. De nombreux paysans utilisent ses feuilles dans les maisons à cause de son odeur qui fait fuir les moustiques. La fumée des feuilles sèches de Cyanotis lanata et de Eucalyptus camaldulensis est hautement insectifuge. Elle est très appréciée par beaucoup de paysans de la province du Kadiogo. II en est de même des feuilles de Corchorus olitorius.

Espèces végétales et techniques de lutte contre les parasites des animaux (les tiques, les poux): Les enquêtes ont permis de répertorier au total treize (13) espèces reparties en douze (12) familles et en treize (13) genres. Les espèces les plus citées dans ce domaine sont : Cymbopogon schoenanthus (73,62\%), Azadirachta indica $(59,72 \%)$, Nicotiana tabacum $(49,57 \%)$, Boswellia dalzielii $(40,09 \%)$, Solanum incanum $(38,21 \%)$, Ricinus communis $(37,32 \%)$ et Cochlospermum planchonii $(32,54 \%)$ (Figure 5). 


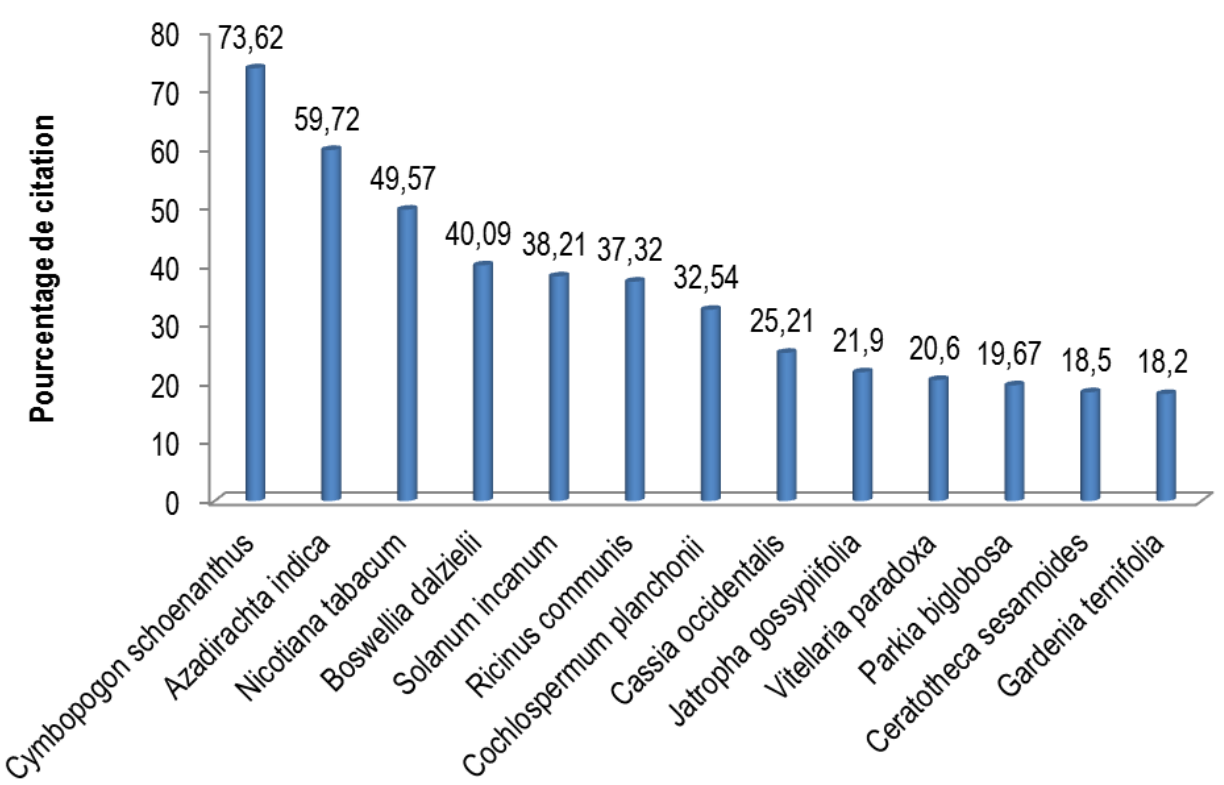

Espèces

Figure 5 : Pourcentage de citation des espèces dans la lutte contre les parasites des animaux

L'écorce rouge de Boswellia dalzielii est utilisée par certains éleveurs ( $40,09 \%$ des enquêtés) de la province du Yatenga pour lutter contre les parasites de la volaille. Cette écorce directement prélevée sur Boswellia dalzielii est déposée à l'endroit où vivent les parasites (poulailler). Ces parasites, attirés par la rougeur de l'écorce viennent pénétrer dans l'écorce, sucent le jus et meurent. Dans d'autres localités, c'est la poudre de tabac qui est beaucoup utilisée pour détruire les parasites de la volaille. Ainsi, la poudre de tabac aspergée ou saupoudrée sur les murs ou sur le sol où vivent les poulets, permet de tuer certains parasites. Ces deux techniques sont beaucoup utilisées par les éleveurs traditionnels. Les feuilles et les inflorescences de Cymbopogon schoenanthus sont également utilisées pour lutter contre les parasites de la volaille. La plupart de nos enquêtés estiment que l'odeur de cette espèce est hautement pestifuge. C'est dans ce sens qu'ils l'utilisent beaucoup pour la confection des nids de poule afin d'y éviter la prolifération d'éventuels pestes. La pâte obtenue après extraction du beurre de Karité (Vitellaria paradoxa) de même que les résidus obtenus après la préparation $\mathrm{du}$ dolo, appliqué au sol ou sur les murs des dortoirs des poulets est hautement pestifuge et pesticide. Cela permet de lutter contre les parasites de la volaille. II en est de même pour la cendre de n'importe quelle plante malaxée dans l'eau et badigeonner au corps des volailles. Pour d'autres habitants, n'importe quelle cendre peut être aussi utilisée pour chasser les termites ravageurs des récoltes. La cendre est simplement utiliser pour saupoudrer le sol sur lequel seront déposées les récoltes. Les feuilles de Cochlospermum planchonii sont utilisées dans la confection des nids de poule, ce qui éviterait la prolifération des pestes. Les feuilles ou les fruits de Cassia occidentalis sont utilisées dans les nids de poulets par certains éleveurs pour lutter contre les tiques. Les feuilles ou de la poudre de tabac, les feuilles de Azadirachta indica, de Ricinus communis ou les fruits de Gardenia ternifolia sont aussi associées aux nids des poules pour prévenir les attaques contre les tiques. Pour prévenir les tiques dans les poulaillers, certains éleveurs associent à l'eau de boisson des poulets, des graines de Solanum incanum. Cette méthode peut aussi être utilisée comme un moyen curatif pour certaines maladies provoquées par les tiques. Des poules ayant consommés les graines de Jatropha gossypiifolia seraient automatiquement immunisées contre les tiques, les poux et les maladies qu'ils provoquent. De la cendre ou de la potasse malaxée dans du beurre de karité et appliquées sur les poulets élimineraient leurs tiques. La potasse liquide est également aspergée aux ailes des poulets pour leur préserver des attaques parasitaires. L'infusion des tiges ou des feuilles de Ceratotheca sesamoides ou de la souffre appliquée sur une tête humaines parasitée par des poux tuerait ces derniers. Certains éleveurs utilisent simplement de la terre mouillée d'eau pour frotter 


\section{Savadogo et al. J. Appl. Biosci. 2016 Méthodes traditionnelles de lutte contre les insectes et les tiques chez}

les Mossé au Burkina Faso

les parties des poulets parasités par des tiques ou des poux. Ces derniers disparaissent en quelques heures. Des feuilles de Parkia biglobosa sont utilisées par quelques éleveurs pour lutter contre les tiques et les ectoparasites de la volaille. Cette méthode consiste à incinérer les dites feuilles dans le poulailler. La fumée qui se dégage serait à mesure de tuer ou de fait fuir les poux et les tiques. De la cendre asperger dans le poulailler ou mouillée d'eau puis aspergée aux poulets présenteraient les mêmes effets. Pour éviter les attaques céréalières par les criquets pèlerins, beaucoup d'agriculteurs vont saupoudrer de la cendre sur les épis de mil. Le beurre de Karité est souvent utilisé par certains éleveurs pour lutter contre les tiques de la volaille. Frotter sur leurs ailes, il empêcherait la prolifération de ses parasites sur les poulets. Pour prévenir les tiques et autres parasites de la volaille, certains éleveurs utilisent également les feuilles sèches de Solanum incanum pour la confection des nids de leurs volailles. L'odeur de ces feuilles empêcherait l'arrivé des tiques dans les poulaillers.

\section{Autres techniques de lutte contre les pestes}

Exposition au soleil: L'exposition des denrées, en couches minces, au rayonnement solaire intense favorise le départ des insectes adultes qui ne supportent pas les fortes chaleurs ni la lumière intense (en stock, les

\section{DISCUSSION}

Les biopesticides jouent incontestablement plusieurs rôles à en croire les populations rurales. C'est pourquoi le (Ministère de l'Environnement et le Cadre de Vie, 2005) souligne que les pesticides contribuent à l'intensification de l'agriculture dans la mesure où ils combattent les divers ravageurs des cultures dont les dégâts peuvent toucher parfois trente (30) à soixante (60) pour cent des produits agricoles. Ils contribuent également à améliorer très sensiblement la santé lorsqu'ils sont utilisés dans la lutte contre les vecteurs des maladies humaines et animales (Ministère de l'Environnement et le Cadre de Vie, 2005 ; RECA, 2013 ; Delimi et al., 2013). La quasitotalité de nos enquêtés est unanime sur les propriétés biopesticides ou biopestifuge des espèces comme Hyptis suaveolens, Hyptis spicigera, Ocimum americanum, Cymbopogon schoenanthus, Anogeissus leiocarpa et Vitellaria paradoxa...Tous ont reconnu les propriétés biopestifuges de ces espèces. Des études antérieures ont également montré l'efficacité des extraits aqueux de Hyptis spicigera, de Euphorbia balsamifera et de Azadirachta indica sur le niébé Vigna unguculata (Bambara \& Tiemtoré, 2008). De nos enquêtes, il ressort que Azadirachta indica n'est pas beaucoup utilisé dans les ménages pour la conservation des céréales et des insectes se cantonnent souvent dans les zones sombres).

Enfumage : Consiste à stocker les grains en épis audessus des foyers domestiques. L'enfumage permanent du lot (parfois appelé à tort fumigation) ne tue pas les insectes mais les éloigne et empêche la réinfestation. Cette méthode est pratiquée sur des épis de maïs, du mil, du sorgho, du riz et des arachides.

Utilisation des matières inertes: Dans des récipients de stockage en vrac (jarres, fûts, greniers) on mélange parfois aux graines de la cendre ou du sable fin, selon des proportions et des pratiques qui varient suivant les régions. Ces matériaux pulvérulents remplissent les vides entre les grains et constituent une barrière à la progression des femelles cherchant à pondre. Ces matériaux fins auraient également un rôle abrasif sur les insectes et entraineraient leur déshydratation. Certains éleveurs utilisent puis asperge dans leurs poulaillers de l'huile de vidange pour empêcher la propagation des tiques ou des poux.

Conservation en atmosphère confinée : Elle consiste à appauvrir en oxygène l'atmosphère inter-granulaire jusqu'à un taux létal pour les insectes. On peut utiliser un silo enterré ou un fût de 200 litres hermétiquement fermé.

légumineuses. $A$. indica étant une espèce exotique, ses vertus biopesticides ne sont pas connues dans beaucoup de localités. C'est surtout dans la région du nord que l'espèce est beaucoup sollicitée pour la conservation des graines du niébé. Le faible taux d'utilisation de $A$. indica serait aussi dû aux mauvaises utilisations de l'espèce qui n'ont pas donné des résultats satisfaisants et, de ce fait, les agriculteurs ont orienté leur choix vers d'autres espèces. Par ailleurs, la monté fulgurante des pesticides de synthèse a beaucoup contribué à un abandon des phytopesticides. En effet, les phytopesticides sont plus difficilement accessibles que les pesticides de synthèse pratiquement disponibles dans tous les marchés. Ce constat sur le terrain nous permet de dire qu'en dépit des multiples travaux réalisés au Burkina Faso sur le Neem, beaucoup de citoyens ignorent toujours ses vertus. Ainsi, les propriétés acaricides et insecticides des formulations biopesticides Neem ont été évaluées en laboratoire et en serre au CRDH sur plusieurs arthropodes causant des dommages économiques importants soient le tétranyque à deux points, le puceron vert du pêcher, l'aleurode des serres et le thrips des petits fruits en laboratoire et en serre (Belanger \& Musabyimana, 2008). Les propriétés insecticides ont été aussi testées avec grand succès en 


\section{Savadogo et al. J. Appl. Biosci. 2016 Méthodes traditionnelles de lutte contre les insectes et les tiques chez les Mossé au Burkina Faso}

plein champ au Burkina Faso sur les haricots verts contre la mouche du haricot et le criquet, sur les pastèques contre la mouche des cucurbitacées, sur la tomate contre la noctuelle de la tomate, les pucerons et les mouches blanches (Belanger et Musabyimana, 2008). Au Québec, les essais en champ ont été effectués sur la fraise (punaise terne), les canneberges (tordeuse des canneberges et pyrale des atocas), le brocoli, le rutabaga, le chou et le radis (mouche du chou), le poivron (punaise terne et pyrale), les oignons (mouche de l'oignon), la pomme de terre (doryphores et pucerons). En République Tchèque, les essaies ont été effectués sur le puceron du choux (Brevicoryne brassicae). Selon certains de nos enquêtés, $A$. indica est utilisé dans plusieurs domaines. En pharmacopée et en cosmétique, I'huile de Neem par exemple est utilisée pour soigner les maux de ventres (vermifuge), le paludisme mais aussi comme répulsif des moustiques. Elle rentre de plus en plus dans la fabrication des savons et des crèmes (Truscott, 2011). Ses activités biopesticides ont été démontrées en Côte d'Ivoire par Diabaté et al (2014).

Trente espèces de plantes au total sont utilisées par les populations à des fins biopesticides ou biopestifuges. Cela montre donc l'importance de la biodiversité dans la lutte contre les pestes. Les savoirs locaux constituent une richesse du point de vue de la diversité biologique. Ils sont considérés d'un point de vue génétique comme de vrais conservatoires de variabilité (Berthaud et Charrier, 1987 , cités par Dupré, 1991). Dans cette optique, la disparition des savoirs locaux équivaut à un appauvrissement du patrimoine génétique, et en définitive, à un amoindrissement des possibilités d'amélioration du matériel végétal. C'est pourquoi Chastanet (in Dupré, 1991) souligne le lien entre pratiques, savoirs et ressources. Elle pense que les savoirs ne se manifestent qu'actualisés dans les pratiques ; la disparition de ces pratiques entraîne la disparition des savoirs sur les ressources (Chastanet, in Dupré, 1991). C'est au regard de son importance que la CDB en son article 8 oblige les États parties à respecter, préserver et maintenir les connaissances, innovations et pratiques des communautés autochtones et locales qui incarnent des modes de vie traditionnels présentant un intérêt pour la conservation et l'utilisation durable de la biodiversité (Yann Le Goater, 2007). Depuis récemment, les savoirs traditionnels sont l'objet de nombreuses recherches scientifiques dans différents domaines. Par exemple, les guérisseurs traditionnels de la forêt tropicale malaise utilisent depuis des temps immémoriaux des extraits d'écorces du Bintagor pour soigner les maux de tête (FAO, 1996). Un chercheur, sur la base d'un échantillon de cette écorce, entreprend des tests en laboratoire et isole le composant chimique pour fournir la base d'un traitement contre certaines formes de cancer (Yann Le Goater, 2007). Beaucoup de plantes utilisées par les agriculteurs pour la conservation des semences sont des plantes aromatiques dont l'efficacité a été démontrée par des études antérieures (Regnault-Toger and Hamraoui, 1997 ; Kellouch et Soltani, 2004). C'est en ayant conscience de cette efficacité des plantes aromatiques qu'un groupement de femmes au Niger, dans le cadre d'un maraîchage en «Bio », ont pu fabriquer un biopesticide composé de tabac, de piment et de neem. Ces productrices de légumes, grâce à ce biopesticide ont eu des résultats satisfaisants dans le domaine du maraîchage (RECA, 2013) bien qu'il ne permet pas de tuer tous les ravageurs. Dans le domaine de la conservation des céréales et des légumineuses, beaucoup de nos enquêtés estiment qu'il est préférables d'utiliser des plantes locales que de faire usage des produits chimiques en ce sens que ces derniers ne sont pas sans conséquences (forte odeur, changement du gout des denrées, coût élevé). Ces préoccupations ont été déjà soulignées par un groupement de femmes productrices de légumes au Niger (RECA, 2013). Pour ces femmes, le produit de traitement à base des plantes ne permet pas de tuer tous les ravageurs mais de limiter les dégâts à un niveau très acceptable. Elles préfèrent un peu de pertes à la manipulation des pesticides trop dangereux à leur goût. De plus elles ne craignent rien quand leurs enfants prélèvent un fruit ou un légume. Aujourd'hui le groupement n'achète plus un seul pesticide chimique. Des études réalisées dans plusieurs agrosystèmes en Afrique et particulièrement au Nord du Cameroun, révèlent que les producteurs utilisent des pratiques traditionnelles dont des extraits des plantes a effet insecticide et/ou insectifuge pour la conservation des produits agricoles en particulier le maïs et le niébé (Ngamo et al., 1981). De par leur innocuité et leur faible toxicité pour l'homme (Isman, SD), ces plantes à utilités alimentaire et médicinale constituent une alternative pour la conservation des denrées stockées. Les activités insecticides des espèces du genre Cymbopogon ont été démontrées par Ketho et al. (2004). Au-delà des plantes, les paysans disposent de multiples moyens pour la conservation des céréales et des légumineuses. II s'agit entre autres de l'exposition des graines de céréales et de légumineuses au soleil, l'enfumage, l'utilisation de matières inertes et la conservation en atmosphère confinée. L'enfumage est surtout beaucoup utilisé par certains paysans car ils l'estiment très efficace. En effet, selon Inge de Groot (2004), la fumée et la chaleur d'un 
feu ordinaire chassent ou tuent les insectes. Cette méthode a certains effets secondaires car le grain continue à sécher et est protégé contre toute réinfestation. Au Togo, plusieurs travaux ont signalé l'existence de ces savoirs traditionnels qui sous-tendent la bonne gestion des ressources phytogénétiques dans différentes régions (Kakabou, 1991 ; Knoth, 1993 ;

\section{CONCLUSION}

Cette étude a permis de connaître les types d'usages traditionnels que les populations font des plantes. Traditionnellement, les plantes sont utilisées dans la conservation des céréales et des légumineuses, la lutte contre les insectes nuisibles à la santé humaine et la lutte contre les parasites de certains animaux. Vingt-quatre espèces au total ont été recensées. Les espèces végétales les plus citées dans la lutte traditionnelle contre les insectes et les tiques sont Hyptis spicigera, Hyptis suaveolens, Azadirachta indica, Calotropis procera, Eucalyptus camaldulensis, Vitellaria paradoxa, Cymbopogon schoenantus, Nicotiana tabacum, Guiera

\section{REMERCIEMENTS}

Nous remercions vivement toutes les populations de la zone d'étude et particulièrement les autorités coutumières des dites zones pour leurs franches collaborations. Nos remerciements vont également à l'endroit des autorités

\section{REFERENCES BIBLIOGRAPHIQUES}

Akpavi S, 2003. Savoirs locaux et gestion des ressources phytogénétiques dans les systèmes de culture dans le Moyen-Mono au Togo. Niamey, CRESA/FA/UAM, $85 \mathrm{pp}$.

Akpavi S, Bamoin M, Batawila K, Vodouhe R, Akpagnak, 2007. Stratégies paysannes de conservation de quelques ressources phylogénétiques dans le moyen-mono au Togo. Agronomie Africaine 19 (3) : 337-349.

Baerts M, Lehmann J, Ansay M, SD. L'usage de plantes en médecine traditionnelle vétérinaire en Afrique sub-saharienne. Hier, aujourd'hui et demain. Élaboration des Pharmacopées 148-166.

Bambara D, Tiemtoré J, 2008. Efficacité biopesticide de Hyptis spicigera Lam., Azadirachta indica A. Juss. Et Euphorbia balsamifera Ait. Sur le niébé Vigna unguculata L. Walp. TROPICULTURA 26 (1) : 53-55.

Belanger A, Musabyimana T, 2008. Le neem contre les insectes et les maladies. Agriculture et Agroalimentaire Canada, Centre de recherche et
Gnamkoulamba, 2001 ; Datchidi, 2002 ; Akpavi, 2003 ; Akpavi et al., 2007). Par ailleurs, dans le sud du Togo, les silos de maïs sont traditionnellement arrosés d'une solution de purin de chèvre (Inge de Groot (2004). L'odeur forte chasse probablement les insectes et même les chèvres qui essaieraient de pénétrer dans le local de stockage.

senegalensis, Khaya senegalensis... Au-delà de ces plantes locales, les paysans, pour lutter contre les pestes font recours à d'autres techniques en l'occurrence l'exposition des graines de céréales et de légumineuses au soleil, l'enfumage, l'utilisation de matières inertes et la conservation en atmosphère confinée. L'acquisition de tous ces résultats permettrait de faire l'économie des méthodes modernes, en l'occurrence les méthodes chimiques qui ont déjà montré leurs effets sur l'environnement; d'où la nécessité de promouvoir et de valoriser ces méthodes traditionnelles.

administratives de l'Institut de Recherche en Sciences Appliquées et Technologies (IRSAT) pour avoir partiellement financer l'étude de terrain.

développement en horticulture, 430 Boul. Gouin, Saint-Jean-sur-Richelieu, Québec, 13 pp.

Benhalima H, Chaudhry MQ, Mills KA, PriceNR, 2004. Phosphine resistance in stored-product insects collected from various grain storage facilities in Morocco. J. Stored Prod. Res. 40: 241-249.

Chandrashekar K, Srinivasa N, 2003. Residual toxicity of selected pesticides, against two spotted spider mites Tetranychus urticae Koch (Acari : Tetranychidae) infesting French bean. J. Ent. Res. 27: 197-201.

Datchidi KM, 2002. Stratégies paysannes de gestion des ressources phytogénétiques cultivées dans la préfecture de Yoto au Togo. Niamey, CRESA/FA/UAM, $71 \mathrm{pp}$.

Delimi A, Taibi F, Fissah A, Gherib S, Bouhkari M, Cheffrour A, 2013. Bioactivité des huiles essentielles de l'armoise blanche Artemessia herba alba : effet sur la reproduction et la mortalité des adultes d'un ravageur des denrées stockées ephestia kuehniella (lepidoptera). Afrique SCIENCE 09(3) : 82-90. 
Diabaté D, Gnago JA, Kofi K, Tano Y, 2014. The effect of pesticides and aqueous extracts of Azadirachta indica (A. Juss) and Jatropha carcus L. on Bemisia tabaci (Gennadius) (Homoptera: Aleyrididae) and Helicoverpa armigera (Hübner) (Lepidoptera: Noctuidae) found on tomato plants in Côte d'Ivoire. Journal of Applied Biosciences 80:7132-7143.

Dupre G, 1991. Savoirs paysans et développement, Paris, Karthala-ORSTOM, 524 pp.

El Guili M, Achbani E, Fahad K, Jijakli H, 2009. Biopesticides : Alternatives à la lutte chimique? Symposium International «Agriculture durable en région Méditerranéenne (AGDUMED)», Rabat, Maroc, 266-280.

Food and Agriculture Organization (FAO), 1996. Malaysia, Country report to the FAO International Technical Conference on Plant Genetic Resources, Kuala Lumpur, FAO Publications, $57 \mathrm{pp}$.

Gnamkoulamba A, 2001. Problématique de la domestication d'une espèce d'igname sauvage : Bayere (Dioscorea praehensilis) à Agbokopé (Préfecture de Wawa au Togo). Niamey, CRESA/FA/UAM, $72 \mathrm{pp}$.

Guèye MT, Seck D, Wathelet JP, Lognay G, 2011. Lutte contre les ravageurs des stocks de céréales et de légumineuses au Sénégal et en Afrique occidentale: synthèse bibliographique. Biotechnologie, Agronomie, Société et Environnement 15 (1):183-194 URL : http://popups.ulg.ac.be/1780-

4507/index.php?id=7108.

Habiba K, 2007. Étude des potentialités d'utilisation d'huiles essentielles pour le contrôle de deux insectes ravageurs des grains Callosobruchus maculatus (Coleoptera : Bruchidae) et Sitophilus zeamais (Coleoptera : Curculionidae) au Nord Cameroun. Thèse de doctorat: Faculté des Sciences, Centre de Recherche sur la Biodiversité, Université Catholique de Louvain (Belgique).

Inge de groot, 2004. Protection des céréales et des légumineuses stockées, Fondation Agromisa, Wageningen, $74 \mathrm{pp}$.

IPNI, 2011. International Plant Names Index database. www.ipni.org.

Isman, SD. Étude des possibilités d'utilisations des formulations à base de fruits secs de Xylopia aethiopica Dunal (Annonaceae) pour la protection des stocks de niébé contre
Callosobruchus maculatus Fabricius (Coleoptera: Bruchidae). Mémoire online, Faculté des Sciences Agronomiques de Gembloux Belgique.

Kakabou B, 1991. Contribution à l'étude des méthodes traditionnelles de conservation des ignames. Lomé, ESA, Univ. Lomé, 53 pp.

Kellouch A, Soltani N, 2004. Activité biologique des poudres de cinq plantes des huiles essentielles, d'une d'entre elle sur Callosobruchus maculatus (F) .Internationale journal of tropicale insecte science 24: 184-191.

Ketho GK, Glitho IA, Koumaglo, 2004. Activité insecticide comparée des huiles essentielles de trois espèces de genre Cympobogon genus (poaceae). J. Soc. Ouest. Afr. Chim 18: 21-34.

Ministère de l'Économie et des Finances, 2010. Schéma national d'aménagement du territoire du Burkina Faso, 93 pp.

Ministère de l'Économie et du Développement, 2006. Atlas du Burkina Faso, 215 pp.

Ministère de l'Environnement et le Cadre de Vie, 2005. Rapport d'inventaires des pesticides pops au Burkina Faso, 75 pp.

Ngamo TLS, Ngassoum MB, Jirovertz L, Ousman A, Nukenin El, Moukala OE, 1981. Protection of stored Maize against Sitophilus zeamais (Motsch.) by use of essential oils of spices from Cameroon. Medical faculty Landbouww University of Gent 66: 473-478.

Knoth J, 1993. Stockage traditionnel de l'igname et de manioc et son amélioration. Eschborn, GTZ, 59 pp.

Noémie L, 2010. Lutte biologique aux ravageurs: applicabilité au Québec. Université de sherbrooke, $103 \mathrm{pp}$.

Ouédraogo E, 2004. L'utilisation des insecticides naturels dans la protection des cultures au Burkina Faso. Communication faite au CTR de I'INERA Ouagadougou CEAS, $56 \mathrm{pp}$.

Ouédraogo S, 2005. Intensification de l'agriculture dans le plateau central du Burkina Faso : une analyse des possibilités à partir des nouvelles technologies. Thèse de Doctorat, Groningen Rijksuniversiteit, 317 pp.

Pintureau B, 2006. Lutte biologique contre les organismes nuisibles à l'agriculture. Futura Sciences, $13 \mathrm{pp}$.

Regnault-Roger C, 2002. De nouveaux phytoinsecticides pour le troisième millénaire ? In : Philogène B.J.R, Regnault-Roger C. \& 
Vincent C., coord. Biopesticides d'origine végétale. Paris : Lavoisier-Éditions Tec \& Doc, 19-39.

Regnault-Roger S, Hamraoui A, 1997. Lutte contre les insectes phytophages par les plantes aromatiques et leurs molécules allelochimiques. Ed Acta Bot.Gallica 144: 401-412.

Réseau National des Chambres d'Agriculture de Niger (RECA), 2013. Le Maraichage en « Bio »: Cela marche chez certaines productrices ou groupements féminins. Note d'information, $4 \mathrm{pp}$.

Sankara E, Namono A, 2004. Analyse institutionnelle des perceptions des principaux acteurs sur les Bonnes Pratiques Agricoles dans les systèmes de production coton céréales-élevage dans l'ouest du Burkina, Bobo-Dioulasso, FAOINERA.

Savadogo S, 2013. Les bois sacrés du Burkina Faso: diversité, structure, dimension spirituelle et mode de gestion de leurs ressources naturelles. Thèse de doctorat unique, Université de Ouagadougou (Burkina Faso), 280 pp.

Sigala $P, 2001$. La lutte contre les pestes végétales sur le domaine forestier à la Réunion. Rev. For. Fr. LIII, 156-162.

Thiombiano A, Schmidt M, Dressler S, Ouédraogo A, Hahn K, Zizka G, 2012. Catalogue des plantes vasculaires du Burkina Faso. Conservatoire et Jardin Botanique de la Ville de Genève. Boissiera, vol 65, ISSN : 0373-2975, 405 pp.

Truscott L, 2011. Textiles Exchange Farm Engagement Newsletter. Engage, $15 \mathrm{pp}$.

Yann Le Goater, 2007. La protection des savoirs traditionnels : l'expérience indienne. Séminaire Jeunes Chercheurs - Association Jeunes Études Indiennes - Aix-en-Provence, 16 pp.

Annexe : Tableau récapitulatif des espèces utilisées et leurs domaines d'utilisation

\begin{tabular}{|l|l|l|l|l|}
\hline Espèces & Partie utilisée & Domaines d'utilisation & Effets & $\begin{array}{l}\text { Nom de l'espèce en } \\
\text { langue locale }\end{array}$ \\
\hline Hyptis spicigera & Feuilles & $\begin{array}{l}\text { Conservation des graines de } \\
\text { niébé et pois de terre }\end{array}$ & Insectifuge & $\begin{array}{l}\text { Younignouga ou } \\
\text { roung-roungui }\end{array}$ \\
\hline Hyptis suaveolens & Feuilles & $\begin{array}{l}\text { Conservation des graines de } \\
\text { niébé et pois de terre }\end{array}$ & Insectifuge & Younignouga \\
\hline $\begin{array}{l}\text { Cymbopogon } \\
\text { schoenanthus }\end{array}$ & Feuilles & $\begin{array}{l}\text { Conservation des semences, } \\
\text { lutte contre les pestes de la } \\
\text { volaille }\end{array}$ & $\begin{array}{l}\text { Insecticide } \\
\text { /Insectifuge }\end{array}$ & Sompiiga \\
\hline Azadirachta indica & $\begin{array}{l}\text { Feuille, graines } \\
\text { et huile }\end{array}$ & $\begin{array}{l}\text { Conservation des semences, } \\
\text { lutte contre les moustiques, } \\
\text { preventions des tiques dans les } \\
\text { poulaillers }\end{array}$ & $\begin{array}{l}\text { Insecticide/ } \\
\text { Insectifuge }\end{array}$ & Nimba \\
\hline Boswellia dalzielii & $\begin{array}{l}\text { Écorce, sève } \\
\text { sèche }\end{array}$ & $\begin{array}{l}\text { Conservation des semences et } \\
\text { lutte contre les moustiques }\end{array}$ & $\begin{array}{l}\text { Insecticide/ } \\
\text { Insectifuge }\end{array}$ & Koumbra yinwougo \\
\hline Anogeissus leiocarpa & Cendre & Conservation des semences & Insecticide & Siiga \\
\hline Vitellaria paradoxa & Cendre & Conservation des semences & Insecticide & Taanga \\
\hline $\begin{array}{l}\text { Solenostemon } \\
\text { rotundifolius }\end{array}$ & $\begin{array}{l}\text { Peau des } \\
\text { tubercules }\end{array}$ & Lutte contre les moustiques & Insectifuge & Pêessa \\
\hline Ocimum americanum & Feuilles & $\begin{array}{l}\text { Conservation des semences, } \\
\text { lutte contre les moustiques }\end{array}$ & $\begin{array}{l}\text { Insecticide / } \\
\text { insectifuge }\end{array}$ & Ninkanssiga \\
\hline Solanum incanum & Fruits & $\begin{array}{l}\text { Lutte contre les parasites de la } \\
\text { volaille }\end{array}$ & Pesticide & Noraog-koumbré \\
\hline Zea mays & Bois de l'épi & Lutte contre les moustiques & Insectifuge & Kamaana \\
\hline Nicotiana tabacum & $\begin{array}{l}\text { Poudre des } \\
\text { feuilles }\end{array}$ & $\begin{array}{l}\text { Lutte contre les parasites de la } \\
\text { volaille }\end{array}$ & Pesticide & Taba \\
\hline Parkia biglobosa & Cendre & $\begin{array}{l}\text { Conservation des graines } \\
\text { d'oseille et pois de terre }\end{array}$ & Insecticide & Roanga \\
\hline Khaya senegalensis & Feuilles & Conservation des céréales & Insecticide & Kouka \\
\hline
\end{tabular}


Savadogo et al. J. Appl. Biosci. 2016 Méthodes traditionnelles de lutte contre les insectes et les tiques chez les Mossé au Burkina Faso

\begin{tabular}{|l|l|l|l|l|}
\hline & & & linsectifuge & \\
\hline $\begin{array}{l}\text { Pterocarpus } \\
\text { erunaceus }\end{array}$ & Écorce & $\begin{array}{l}\text { Lutte contre les dermatoses } \\
\text { buccales des animaux }\end{array}$ & Pesticide & Noega \\
\hline Cyanotis lanata & Feuilles sèches & Lutte contre les moustiques & Insectifuge & Saninsaanga \\
\hline $\begin{array}{l}\text { Eucalyptis } \\
\text { camaldulensis }\end{array}$ & Feuilles sèches & Lutte contre les moustiques & Insectifuge & Eucalyptus \\
\hline Eleusine indica & Feuilles & Lutte contre les moustiques & Insectifuge & Targanga \\
\hline Capparis sepiaria & Feuilles pilées & $\begin{array}{l}\text { Conservation de n'importe } \\
\text { quelle semence }\end{array}$ & Insecticide & Galingooré \\
\hline Corchorus olitorius & Feuilles sèches & Lutte contre les moustiques & Insectifuge & Boulvanka \\
\hline Ricinus communis & Feuilles & $\begin{array}{l}\text { Prévention des tiques dans les } \\
\text { poulaillers }\end{array}$ & Pestifuge & Ricinus \\
\hline Gardenia ternifolia & Fruits & $\begin{array}{l}\text { Prévention des tiques dans les } \\
\text { poulaillers }\end{array}$ & Pestifuge & Rambrizounga \\
\hline Cassia occidentalis & Fruits ou feuilles & $\begin{array}{l}\text { Prévention des tiques dans les } \\
\text { poulaillers }\end{array}$ & Pestifuge & Katr nangouri \\
\hline $\begin{array}{l}\text { Ceratotheca } \\
\text { sesamoides }\end{array}$ & Feuilles broyées & $\begin{array}{l}\text { Prévention des attaques par les } \\
\text { poux, les tiques des animaux }\end{array}$ & Insecticide & Boundou \\
\hline
\end{tabular}

